\title{
Stick-slip behavior of ice streams: modeling investigations
}

\author{
Olga V. SERGIENKO, ${ }^{1 *}$ Douglas R. MacAYEAL, ${ }^{2}$ Robert A. BINDSCHADLER ${ }^{3}$ \\ ${ }^{1}$ Department of Geology, Portland State University, 1721 SW Broadway, Portland, OR 97201, USA \\ E-mail: osergien@princeton.edu \\ ${ }^{2}$ Department of Geophysical Sciences, University of Chicago, 5734 South Ellis Avenue, Chicago, IL 60637, USA \\ ${ }^{3}$ Hydrospheric and Biospheric Science Laboratory, NASA Goddard Space Flight Center, Greenbelt, MD 20771, USA
}

\begin{abstract}
A puzzling phenomenon of ice-stream flow is the stick-slip motion displayed by Whillans Ice Stream (WIS), West Antarctica. In this study we test the hypothesis that the WIS stick-slip motion has features similar to those of other known stick-slip systems, and thus might be of the same origin. To do so, we adapt a simple mechanical model widely used in seismology to study classic stick-slip behavior observed in tectonic faults, in which the difference between static and dynamic friction allows for the generation and spatial propagation of abrupt slip events. We show how spatial variability in friction properties, as well as a periodic forcing intended to mimic the effect of tides, can reproduce the observed duration and periodicity of stick-slip motion in an ice stream. An intriguing aspect of the association of WIS with mechanical stick-slip oscillators is that the onset of stick-slip cycling from a condition of permanent slip appears to be associated with the reduction in overall speed of WIS. If this association is true, then stick-slip behavior of WIS is a transitional phase of behavior associated with the ice stream's recent deceleration.
\end{abstract}

\section{INTRODUCTION}

The term stick-slip refers to the motion of systems where elastic forces modulate the sliding of rigid objects over substrates that exhibit the classic difference between static and kinetic coefficients of friction, and applies to situations readily encountered in everyday life (e.g. when automobile tires screech, when door hinges squeak, when stringed instruments are bowed and when heavy objects are dragged across rough floors). The key property of stick-slip motion is that the kinematics is divided into two phases: a 'static' phase where the object is 'stuck', and a 'kinetic' phase where the object is 'slipping'. This phenomenon is observed in a wide range of natural systems, and may be the simplest fundamental way to describe seismic and coseismic movements associated with earthquakes at plate boundaries of the Earth's crust. The single most important physical property shared by stick-slip systems is that the magnitude of the static friction is larger than the magnitude of the kinematic friction. A body at rest starts to move relative to a frictional substrate only when the net force exerted on it exceeds a resisting static friction. Once in motion, the same body will experience reduced friction (kinetic friction) until its motion relative to the substrate ceases and the body again becomes stuck by the larger static friction.

In the glaciological world, a phenomenon that resembles stick-slip is observed in the flow of several outlet glaciers (Chandler and others, 2005; Danesi and others, 2007), and just one (so far) of the West Antarctic ice streams, Whillans Ice Stream (WIS, former Ice Stream B) (Bindschadler and others, 2003a; Joughin and others, 2005; Wiens and others, 2008). As revealed by global positioning system (GPS) measurements, rapid horizontal displacements $(\sim 0.5 \mathrm{~m})$ of a large portion of the ice stream near its grounding zone occur once or twice per day. These rapid slip events span a

*Present address: Princeton University/GFDL, 201 Forrestal Road, Princeton, NJ 08540, USA. relatively short time interval $(\sim 30 \mathrm{~min})$ and are separated by long periods of more-or-less steady flow, yielding a similar net inter-slip-event displacement of $\sim 0.5 \mathrm{~m}$ (Fig. $1 \mathrm{~b}$, c, e and $f$.

The cause of stick-slip cycling on WIS has been debated. Bindschadler and others $(2003 a, b)$ have suggested that it is a result of an interaction of a plastic nature of the till underlying WIS and a tidal forcing in the open Ross Sea immediately downstream of the ice stream. Indeed, all GPS measurements collected on WIS, as well as those on other active ice streams, have displayed strong tidal signals in the horizontal flows of the ice streams (Anandakrishnan and others, 2003; Gudmundsson, 2007), but, except for WIS, these signals are smoothly varying in time. Weertman (2005) has argued that stick-slip is caused by subsonic propagation of gliding edge dislocations at the ice/till interface. These dislocations cause lower water pressure in the till, that subsequently attracts water to the ice/till interface and causes abrupt sliding. This explanation does not involve specific characteristics of the till or its deformation, and rather puts an emphasis on the subglacial water stored in the till and lowered hydrostatic pressure that draws water to the ice/till interface. A recent study involving seismological signals associated with the stick-slip flow of WIS (Wiens and others, 2008) has proposed that stick-slip behavior results from a sticky spot in the icestream bed. They argue that the ice stream is locked in the vicinity of this sticky spot until stress build-up induces a slip event, which generates seismic radiation observed teleseismically (e.g. at the South Pole). Associated with the seismic signal is the $\sim 0.5 \mathrm{~m}$ coseismic displacement of the ice stream that begins on top of the sticky spot, and propagates to the neighboring region at relatively fast speeds ( 500-1000 $\left.\mathrm{m} \mathrm{s}^{-1}\right)$.

The objectives of this study are: (1) to determine whether WIS represents a typical stick-slip system in which motion is controlled by the same fundamental properties as the most simple stick-slip systems described by the idealized mechanical model; (2) to determine whether a simple 

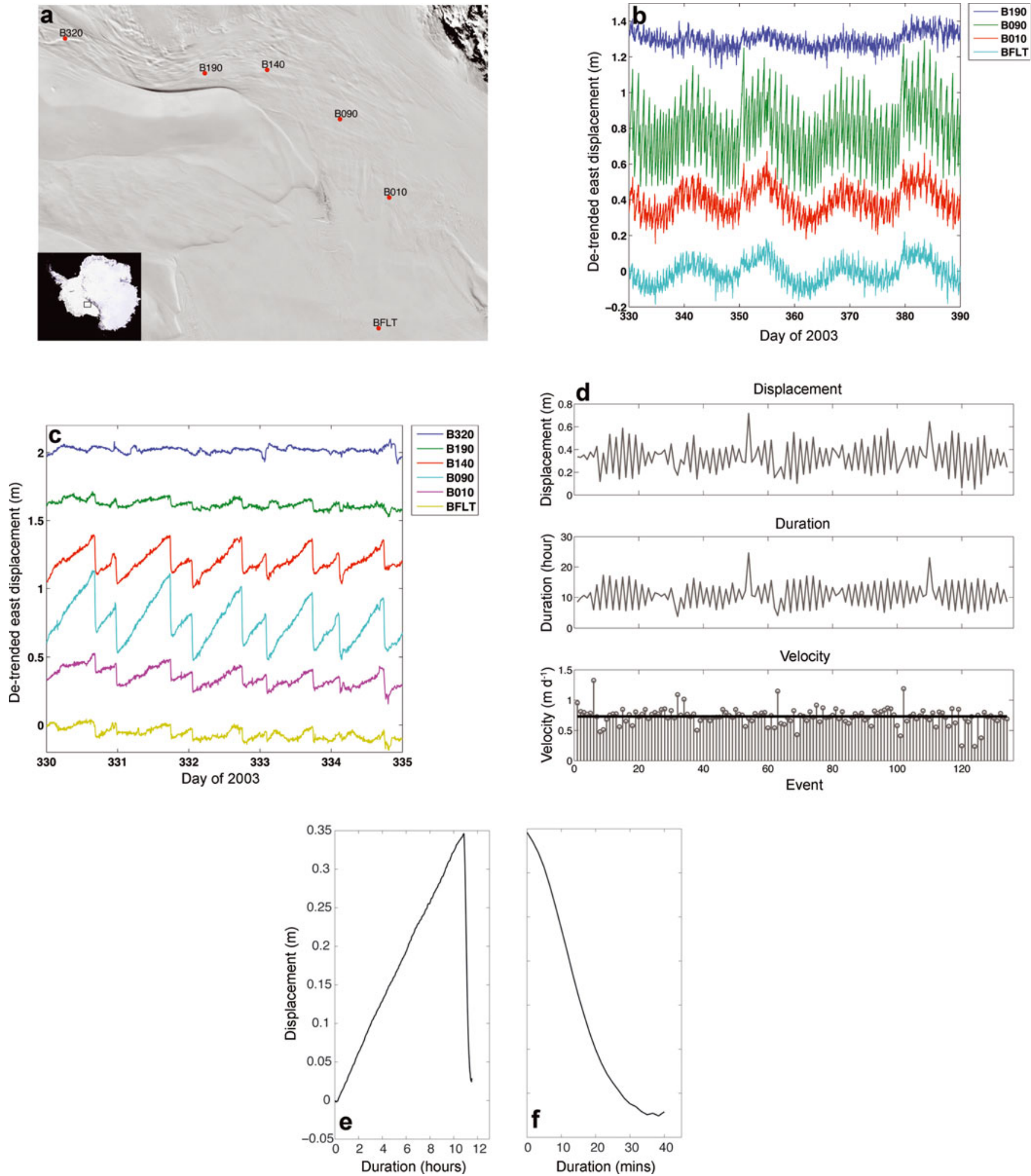

Fig. 1. Observations of stick-slip events on WIS. (a) MODIS (moderate-resolution imaging spectroradiometer) image of WIS and part of the Ross Ice Shelf, showing locations of GPS stations used to reveal stick-slip behavior. Inset is a Mosaic of Antarctica (MOA) image of Antarctica. (b) 30 day detrended east-west displacement. (c) 5 day detrended east-west displacement. (d) Characteristics of stick events observed by GPS station B090. Black line on the velocity panel indicates mean velocity value $0.72 \mathrm{~m} \mathrm{~d}^{-1}$. Displacements and durations are in phase with spring-to-neap amplitude variation of the diurnal tide. (e) Close-up of typical stick-slip events determined by stacking, stretching and averaging multiple observed cycles. (f) Close-up of the slip phase shown in (e).

mechanical model can explain the observed features of the WIS stick-slip motion described below; and (3) to gain a basic understanding, since the model is extremely idealized, of why WIS exhibits stick-slip behavior while other ice streams appear (so far) to flow in a continuous manner. To meet these objectives we adapt an idealized mechanical model widely used in earthquake fault studies (e.g. Burridge and Knopoff, 1967; Carlson and Langer, 1989) and qualitatively compare its results with the WIS GPS observations. 


\section{OBSERVED FEATURES OF THE WIS STICK-SLIP CYCLE}

The basic approach undertaken in this study is to investigate the observed stick-slip phenomena of WIS using an idealized model. To this end, it is appropriate to describe the basic phenomenological features of WIS's stick-slip motion, to serve as a list of performance constraints to which the idealized model will be subject. As Figure $1 \mathrm{~b}$ and c shows, the WIS flow has strong variability, both temporal and spatial. Despite this variability, we can distinguish a pattern that allows us to 'idealize' the WIS flow in the following manner: (1) Stick-slip events occur only on the ice plain immediately upstream of the grounding zone. Station B320, for example, is located near onset of WIS far from the ice plain, and its GPS record does not show stick-slip (Fig. 1). (2) During the static period between slip events, the ice stream flows at a much slower rate than during the slip phase of the cycle. Analysis of the displacements and durations during the static phase of WIS's motion recorded at station B090 shows a flow of $90 \mathrm{~m} \mathrm{a}^{-1}$, which compares with $\sim 8000 \mathrm{~m} \mathrm{a}^{-1}$ during the slip phase $(5 \mathrm{~min}$ Precise Point Positioning (PPP) obtained from GPS data is not sufficient to perform a similar analysis for slip amplitude and duration, and to obtain a better estimate for ice speed during the slip phase). (3) Most importantly, transitions between stick and slip phases appear to have several distinctive properties (Bindschadler and others, 2003a; Wiens and others, 2008) whose origins constitute key objectives of this study:

Slip starts, or nucleates, at a single, unchanging location before slip starts elsewhere.

Amplitude of displacement during the slip phase decays upstream from this nucleation site, and is zero far downstream, near the calving front of the Ross Ice Shelf (MacAyeal and others, 2007) and far upstream, near the ice-stream onset (i.e. the flow is continuous far upstream and far downstream).

Slip events display irregular periodicity, and typically occur in doublets, where second slip events (aftershocks) follow primary, periodic, slip events, but often at irregular following time intervals (Fig. 1C).

The time duration and amplitude of stick phases are strongly correlated with the spring-to-neap cycle of the ocean tide (Fig. 1d).

The stick phase lasts 10-30 times longer than the slip phase (Fig. 1e-f).

A reasonable approach to beginning the task of understanding the causes of the above features is to examine whether the simplest models capable of representing stickslip systems, i.e. block-and-spring models, might also feature the above idealized properties. This is the motivation for introducing the idealized mechanical model that follows.

\section{AN IDEALIZED BLOCK-AND-SPRING MODEL OF AN ICE-STREAM STICK-SLIP PHENOMENON}

We adapt a simple mechanical analogue model, consisting of rigid blocks connected by springs, that is widely used for stick-slip studies in other geophysical contexts (e.g. Carlson and Langer, 1989). As displayed in Figure 2a, rectangular blocks of uniform mass, $m$, and uniform dimensions, i.e. area of basal contact, are connected by pairs of springs, with stiffness $\lambda / 2$, to a pair of long rigid blocks on each side, and to each other by springs with stiffness $k$. Each block rests on a lower surface that moves with constant speed, $V$. A reference frame is adopted that moves with this steady flow, so the subglacial bed, represented by the lower surface, moves at a rate, $V$, opposite to the direction of ice flow. The sequence of small blocks in between the two long blocks on the left and right represent the part of the ice stream that participates in a stick-slip cycle. The springs represent elastic connections between the small blocks and the surrounding ice stream, and between the small blocks themselves.

The basic model set-up and the investigation of the singleblock experiment, detailed below, follow the original paper by Carlson and Langer (1989), and are intended to guide a plausible choice of parameter values that must apply if the model does describe the stick-slip motion of WIS. The tidal single-block experiment, below, follows previous work by Bindschadler and others (2003a), which we expand to investigate the effect of a spatially extended, inhomogeneous system on slip timing, duration and periodicity.

We choose, in this study, to restrict our attention to the behavior implied by the most simple treatment of friction, so the friction force, $F$, acting on each block can have one of two magnitudes, either $F=F_{\mathrm{s}}$ or $F=F_{\mathrm{k}}$, where $F_{\mathrm{s}}$ and $F_{\mathrm{k}}$ are constants. The choice of which constant $F$ is equal to depends on whether the block is sliding across the lower surface (during the kinetic, or slip, phase) or is motionless relative to the lower surface (the static, or stick, phase). A static friction, $F_{\mathrm{s}}$, applies when the relative velocity between a block and the lower surface is zero, and a velocity-independent sliding (or kinematic) friction, $F_{\mathrm{k}}$, applies when a block slides across the lower surface. This is the simplest frictional law that describes the contact of two bodies, and is one that is familiar to all physics students. This law is notably more simple than the typical Coulomb (e.g. Schoof, 2006b) or plastic (e.g. Tulaczyk and others, 2000) friction laws commonly used in glaciology, which express $F_{\mathrm{k}}$ in terms of normal stress, cohesion, water pressure and shear stress (e.g. Rice and Tse, 1986; Afferrante and Ciavarella, 2008). (An extensive and comprehensive description of rate- and state-dependent friction laws is given by Rice and others (2001); detailed mathematical treatment of Coulomb friction properties can be found in Schoof (2006b,a).) It is important to keep in mind, however, that despite the simplicity of a system which has only two friction force magnitudes, the transitions between static and kinetic phases are complex. The decision-making rules necessary for specifying the transitions between the two phases are summarized in Figure 2b.

The equations of motion of the $j$ th block in the sequence of blocks depicted in Figure 2a are

$$
\begin{array}{r}
\dot{X}_{j} \equiv u_{j}=V \quad \text { if }\left|k\left[X_{j+1}-2 X_{j}+X_{j-1}\right]-\lambda X_{j}\right| \leq F_{\mathrm{s}} \\
m \dot{u}_{j}=k\left[X_{j+1}-2 X_{j}+X_{j-1}\right]-\lambda X_{j}-F_{\mathrm{k}} \operatorname{sgn}\left(u_{j}-V\right) \\
\text { if } u_{j} \neq V,
\end{array}
$$

where $X_{j}$ is the position of the $j$ th block, $u_{j}$ and $\dot{u}_{j}$ are its velocity and acceleration, respectively, and $\operatorname{sgn}(v)$ denotes the sign function that equals -1 when its argument is less 


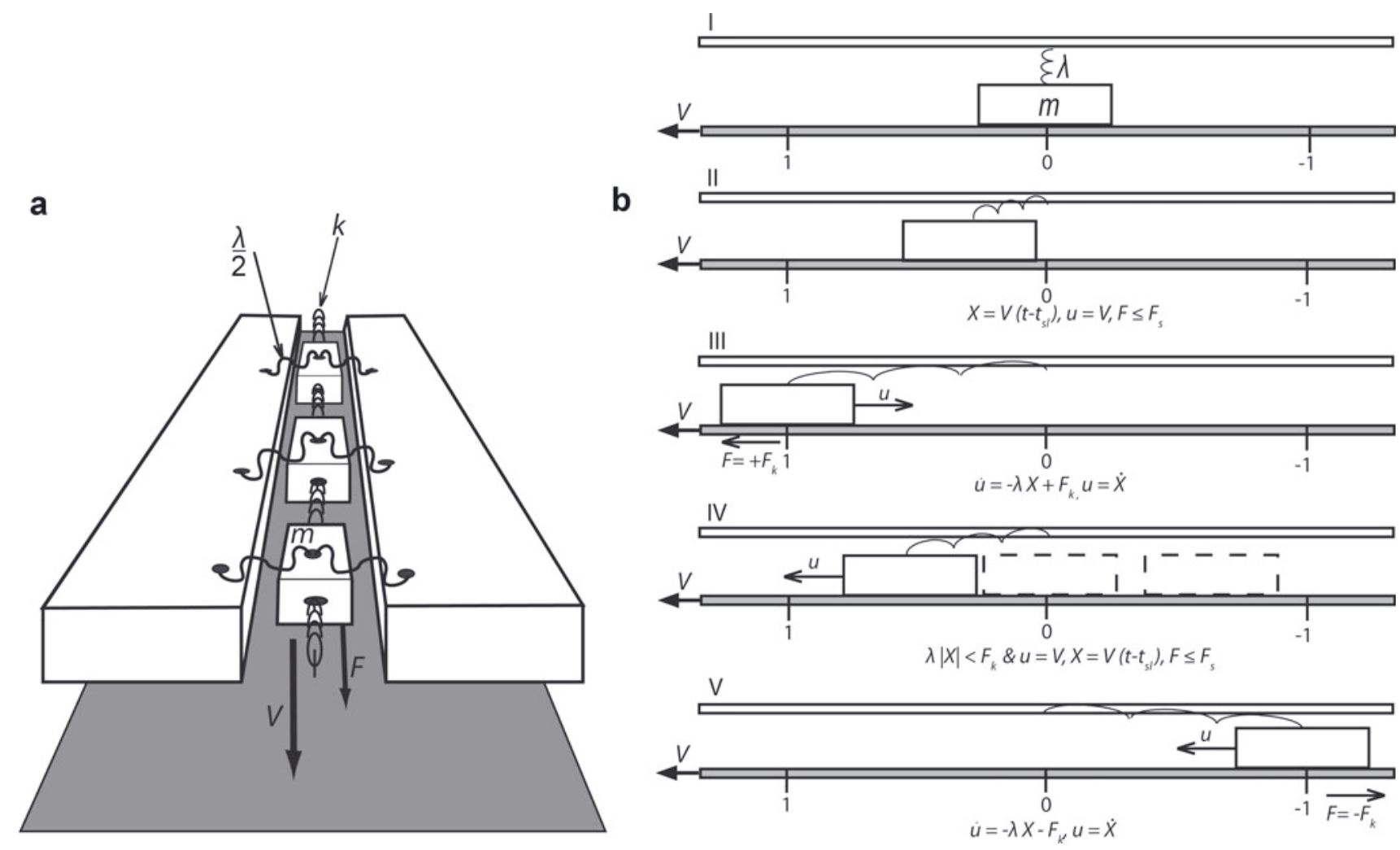

Fig. 2. Idealized mechanical analogue model of an ice stream capable of stick-slip motion (adapted from Carlson and Langer, 1989). (a) A system of uniform blocks connected by springs designed to mimic an ice stream. (b) A single-block system at various stages: I-II. 'stick' phase; III. 'slip' phase (starting when the block reaches a position where the spring reaches maximum extension); IV. end of 'slip' phase and beginning of a 'stick' phase (dashed blocks illustrate various intermediate positions); $\mathrm{V}$. in the case when $F_{\mathrm{k}}$ is sufficiently low, the block can slide to a limiting position of the spring extension in the opposite direction.

than zero and 1 if its argument is greater than zero. Initial conditions are such that relative displacements of all blocks are 0 , i.e. $X_{j}=0$ and their velocities $u_{j}=V$.

Equation (1) describes the static, or stick, phase of the motion of a block: the particular block is stuck to the lower surface, and is thus retarded relative to the motion of the 'ice stream proper' represented by the two long blocks on either side. As the static phase progresses, the increasing relative displacement between the block and the ice stream proper causes the combined spring force (with total spring elasticity, $\lambda$ ) to eventually overcome the static friction, $F_{\mathrm{s}}$. Once this happens, the block transits from the static phase to the slip phase of behavior that is described by Equation (2). While the block is sliding, the spring forces resisted by kinematic friction cause the block to rebound, and to catch up with the ice stream proper. Eventually, the block's motion is slowed by spring forces and kinetic friction to the point where its motion relative to the substratum again becomes zero. When this happens, the block leaves the kinetic phase and re-enters the static phase of its cyclic stick-slip motion.

\section{MODEL EXPERIMENTS}

Using various scenarios we explore the ability of the abovedescribed mechanical analogue model to resemble the features of the WIS stick-slip phenomena outlined in the previous section. We idealize the portion of the ice stream undergoing stick-slip cycling as either a single block, or as a sequence of ten blocks (representing a longitudinal section of the ice stream). The set of ordinary differential
equations(ODEs), Equations (1-2), representing the array of ten blocks is solved using the Matlab ${ }^{T M}$ ODE solver, ode45, based on the explicit fourth-order Runge-Kutta algorithm. (We checked the performance of this solution approach by also coding a much simpler finite-difference version of the second time derivatives in Equation (2), and found the results to be nearly identical.) All experiments are done with $V=0.1$ and $m=1$. The choice of parameters $\lambda, k, F_{\mathrm{s}}$ and $F_{\mathrm{k}}$ is explained below.

Each of the following subsections represents an experiment designed to mimic the behavior of this system. To obtain a basic understanding, and keep experiments simple, only one parameter is varied for each experiment.

\section{One-block system}

The first set of experiments is done with a single-block system. This system is identical to the behavior of a tenblock system when $F_{\mathrm{s}}, F_{\mathrm{k}}$ and $\lambda$ are the same for all blocks. The one-block system is used to determine an appropriate range of parameters leading to behaviors that are similar to those of the WIS, and to gain insight into what effects may be produced once parameters are allowed to vary from block to block. In this analysis we follow Burridge and Knopoff (1967) and Carlson and Langer (1989). The maximum block displacement relative to the ice stream proper is achieved at the end of a stick phase (Fig. 2b, III), and is determined by the condition when the spring forces equal the static friction,

$$
X_{0}=\frac{F_{\mathrm{s}}}{\lambda}
$$



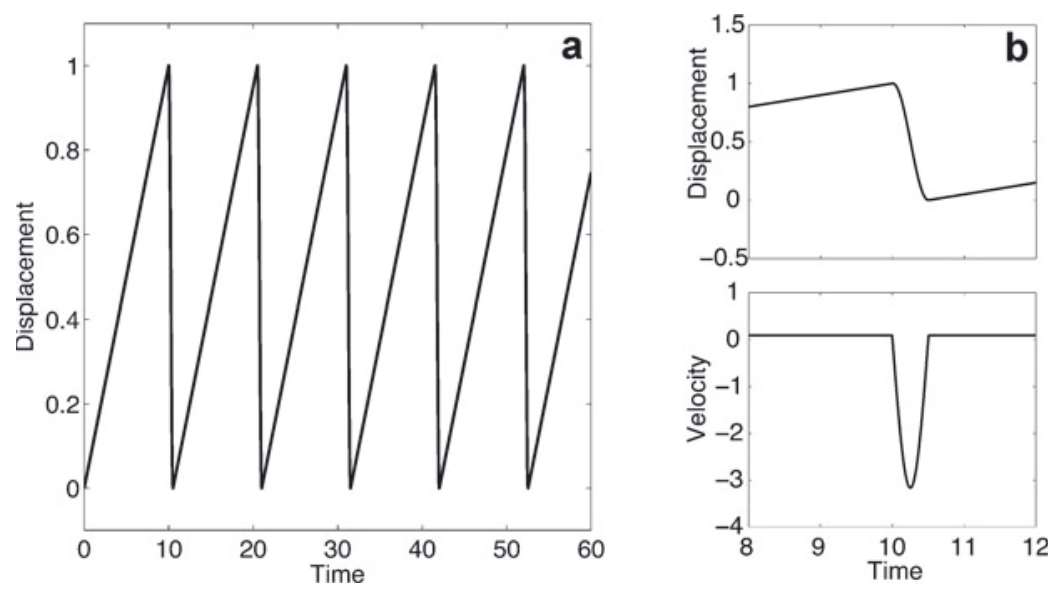

Fig. 3. Stick-slip behavior of a homogeneous system. (a) Displacement; (b) close-up of a slip event. The slip phase is of 20 times shorter duration than the stick phase.

The maximum block displacement in the slip phase (in the direction opposite to the motion of the lower surface) is determined by the ratio of the kinetic to static friction (Fig. 2b, IV). If the ratio is close to 1 , the block's rebound during the slip phase is relatively small and the block resticks to the substrate relatively soon after beginning to slide. In the case where $F_{\mathrm{k}} / F_{\mathrm{s}}<0.5$ the block is able to surge ahead of the ice stream proper, thereby overshooting the ice stream before entering a static phase. If $F_{k}$ is small enough, the block exhibits elastic oscillations, and slides back and forth before re-sticking to the lower surface. The coseismic displacements of the part of WIS undergoing strongest stickslip cycles do not exhibit such oscillations (i.e. where the part of the ice stream originally stuck becomes wobbly, like a mass of gelatine), and this condition implies that $F_{\mathrm{k}} / F_{\mathrm{s}} \geq 0.5$. In the case where $F_{\mathrm{k}}=0.5 F_{\mathrm{s}}$, the block just catches up to its equilibrium position, $X=0$, at the end of its slip phase. This ratio is chosen for the rest of the experiments described below.

Under these circumstances the duration of the stick phase is given by

$$
\tau_{\mathrm{st}}=\frac{X_{0}}{V}=\frac{F_{\mathrm{s}}}{\lambda V}
$$

and the duration of the slip phase, $\tau_{\mathrm{s} \text { l, }}$ is primarily determined by the spring constant, $\lambda$. The block's velocity relative to the ice stream proper is $u-V$, and is proportional to $\sin (\sqrt{\lambda} t)$, i.e. it varies as half of a sine wave (we assume no reverse motion, $F_{\mathrm{k}} / F_{\mathrm{s}} \geq 0.5$ ), so $\sqrt{\lambda \tau_{\mathrm{s}}}=\pi$. The slip duration observed on WIS is at least an order of magnitude shorter than the duration of the stick phase. We thus conclude that $\sqrt{\lambda} \approx 10 \div 30\left(\pi / \tau_{\mathrm{st}}\right)$. For our simulations, we choose $\tau_{\mathrm{st}} / \tau_{\mathrm{sl}} \approx 20$ and $\lambda=F_{\mathrm{s}}$ to give $\lambda=F_{\mathrm{s}}=40$.

Figure 3 displays the time-dependent behavior of the single-block (homogeneous) system with the parameter ranges chosen above. The model set-up and the resulting simulations resemble those described by Carlson and Langer (1989). The system is capable of producing regular stick-slip events with the ratio of stick to slip time durations observed on WIS. As Figure 1d shows, both displacement and duration of stick events are strongly correlated with spring-to-neap ocean tide cycles. To attempt to simulate this kind of tidal effect in our simple model, we apply a periodic forcing to the system (i.e. an additional force, $F_{t}(t)$, is introduced on the right-hand side of Equation (2)) with a time variability that mimics the spring-to-neap tidal cycle (top curve in Fig. 4b):

$$
F_{\mathrm{t}}(t)=A(\sin \omega t+\sin 1.079 \omega t) .
$$

The coefficient 1.079 is the ratio of the periods of the two diurnal $\mathrm{O} 1$ and $\mathrm{K} 1$ tidal components that are dominant in the Ross Sea. Figure 4 shows the displacement of a single block in response to $F_{\mathrm{t}}(t)$ with parameters $A=10$ and $\omega=2 \pi / 20$ (the choice of these parameters is justified below). Two features become apparent in the tidally forced stick-slip cycle: (1) a second slip event, or aftershock, is present during spring phase of the forcing (Fig. 4a), and (2) the displacement amplitude has long-period variations that follow the amplitude envelope of the forcing, $F_{\mathrm{t}}(t)$. These two features of the simple model are similar to observed features of WIS shown in Figure $1 \mathrm{~b}$ and $\mathrm{c}$. The doublet structure of slip events (this term is used because two events occur during one cycle of the carrier, or tidal, frequency, $\omega$ ) is sensitive to the choice of parameters, both the amplitude, $A$, and the frequency, $\omega$, of the carrier phase of the forcing. Lower $\omega$ tends to produce more frequent aftershock slip events. For example, choosing $\omega$ to be half the value specified above results in triplet slip events for each period of the carrier signal. The doublet structure is produced in a range of forcing amplitudes $10-50 \%$ of $F_{\mathrm{s}}$. Outside that range, the doublet structure disappears.

As the results show, the single-block system can explain several of the phenomenological features of the stick-slip cycle observed on WIS, listed above. Specifically, the simple model is capable of motions that match the following features of the WIS cycles: (1) the ratio of the stick and slip phase time durations, (2) the doublet structure during tidal cycles, and (3) long-period variations of the displacement amplitude associated with amplitude modulation (springto-neap) of tidal cycles. A strong sensitivity of the stick-slip doublet structure to the parameters of the external forcing suggests that it is an intrinsic feature of the block-and-spring system (or ice stream), and not a feature of the timedependent tidal forcing, and is mostly determined by the static friction, $F_{\mathrm{s}}$, and the spring constant, $\lambda$. These results are consistent with the analysis and interpretation presented by Bindschadler and others (2003a). 

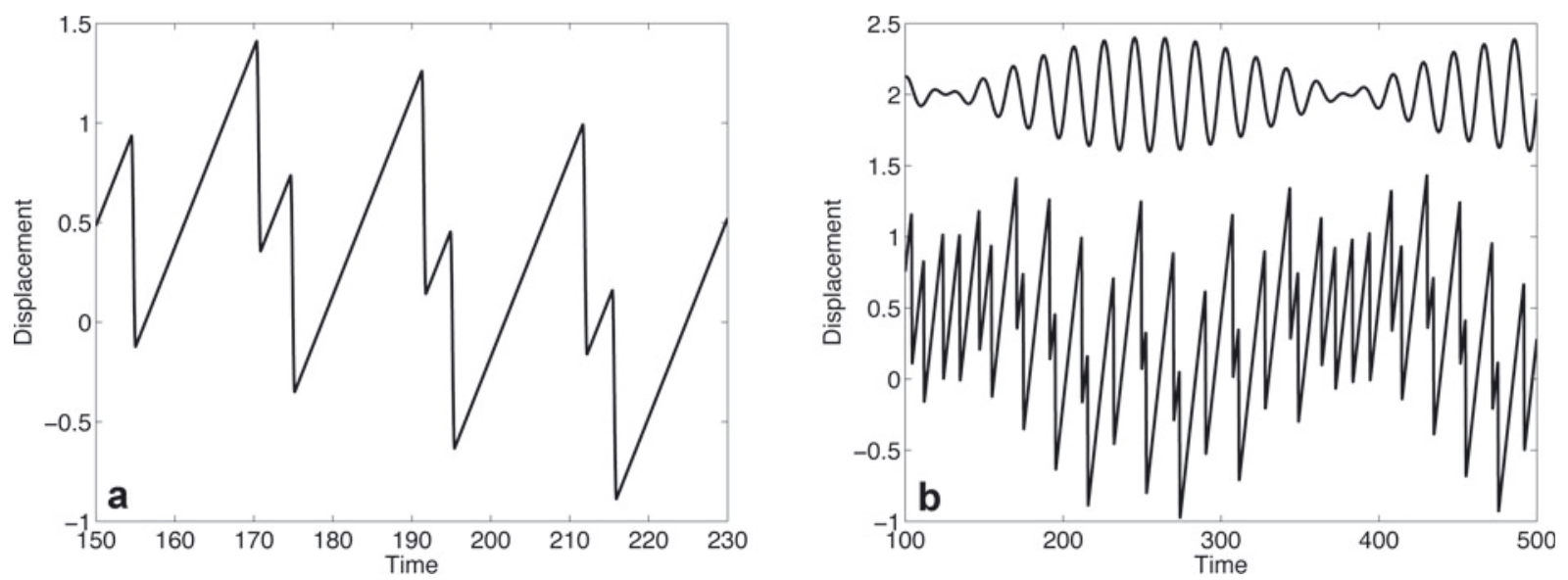

Fig. 4. Effect of a 'spring-to-neap' tide. (a) Development of a secondary slip event ('doublet'). (b) Periodic variations in amplitude due to a periodic forcing, $F_{\mathrm{t}}(t)$ (top curve).

\section{Heterogeneous system}

The features of the WIS stick-slip motion associated with spatial variability (e.g. the decay of stick-slip amplitude with distance from the sticky spot on the ice stream) cannot be explained with the single-block system. To explore the cause of this variability, we introduce heterogeneity by allowing more than one block in the simple model (as shown in Fig. 2a), and allowing the friction parameters to vary from block to block. Since the system is not homogeneous, the block displacements are not the same, and this causes the springs connecting the blocks to each other to start to stretch. This inter-block spring stretching introduces additional forcing into the system and results in additional complexity of the stick-slip motion.

The first experiment is designed to mimic tidal effects. A periodic forcing, $F_{\mathrm{t}}(t)$ (Equation (5)), is applied to the last block of a ten-block system. In this experiment, the kinetic and static friction parameters are equal for all blocks. The result shows that the amplitude of the stick-slip events decays with the distance from the block that is forced (Fig. 5a); this is similar to observations on WIS which show decay of stickslip motion upstream from the grounding line.

To investigate the effects of a sticky spot, such as proposed by Wiens and others (2008), we increase the friction parameters, $F_{\mathrm{s}}$ and $F_{\mathrm{k}}$, of one block (block number 5 , midway between the upper end and the lower end of the longitudinal sequence of blocks) by $50 \%$ of their original values. As Figure $5 \mathrm{~b}$ shows, the blocks slip by various distances and with various frequencies depending on how close to the center of the block sequence they are (i.e. how close to the 'sticky spot'). The amplitude of the motion is largest for the block with increased friction, and decreases for blocks located farther from the 'sticky spot'. This decay illustrates a phenomenological feature of the real WIS, and suggests that the proposition by Wiens and others (2008) that a sticky spot is essential to the stick-slip motion of WIS is indeed reasonable.

\section{Initiation of stick-slip motion}

Here we investigate the transitional behavior of the mechanical model, from a stable sliding mode to a stick-slip mode, as a means of exploring why WIS appears to possess stickslip behavior, unlike all the other ice streams studied so far. To do that, we again use the homogeneous (single-block) system described above. The results are best interpreted by considering that the characteristic time, $\tau$ (Equation (4)), required for the block to reach maximum displacement,
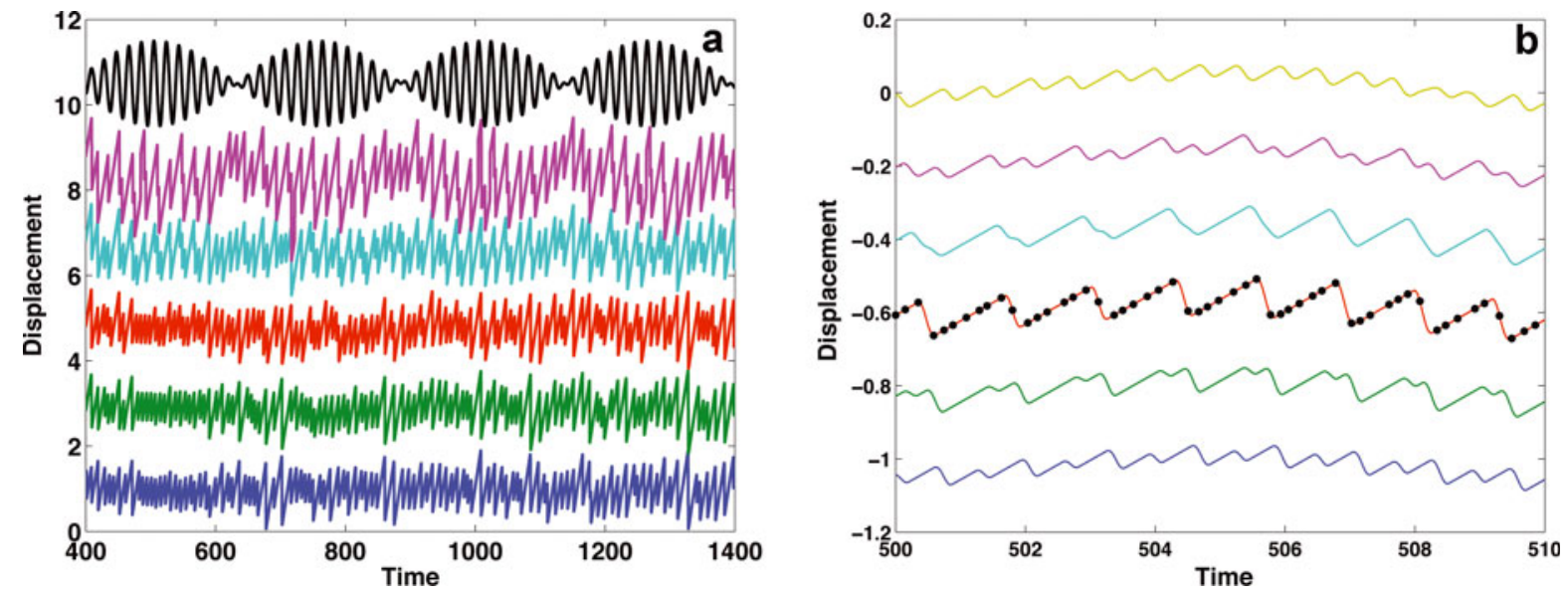

Fig. 5. Heterogeneous system. (a) 'Tide' effects. Top black curve shows applied forcing. Amplitude decreases with the distance from the applied forcing. (b) 'Sticky spot' effect. Dashed red line indicates location of the 'sticky block' relative to other blocks. Double slip events develop upstream and downstream of the sticky spot. 
$X_{0}$ (Equation (3)), can be thought of as a parameter that determines whether stick-slip will occur at all. Two limiting cases, permanent stick and permanent slip, correspond to $\tau=\infty$ and $\tau=0$, respectively. Transition from the permanent slip mode to the stick-slip mode is equivalent to requiring $\tau$ to increase from 0 to a finite value. As Equation (4) shows, this can be achieved either by slow, secular increases in $F_{\mathrm{s}}$ or by slow, secular decreases in $V$. Initiation of stick-slip motion in a decelerating mechanical system (i.e. decreasing $V$ at a rate that is slow compared to the motion during individual cycles) was experimentally observed by De Baets and others (2000).

There are numerous reports that WIS has been slowing since the early $1960 \mathrm{~s}$ at a rate of $\sim 2-4 \mathrm{ma}^{-2}$ (e.g. Bindschadler and Vornberger, 1998; Joughin and others, 2005). This deceleration is attributed to changes in the availability of subglacial water and associated strengthening of the till, implying that basal friction below WIS is increasing. Since ice streams are complicated systems where one factor can affect many others, it is difficult to gauge whether the increase in basal friction results from a consequent ice-flow speed reduction or vice versa. Most likely, however, both factors have contributed (or reinforced each other) to cause the WIS system to approach a transition from steady sliding (like other ice streams) to a stick-slip regime.

The results of the experiments described here and observational evidence suggest that further WIS deceleration would be accompanied by an increase in the period of cyclic stick-slip events on the ice stream. This could perhaps be tested observationally. Whether WIS will eventually approach another transition, i.e. to a mode of permanent stick, such as is the mode of Kamb Ice Stream, West Antarctica, will depend on whether the basal friction parameters continue to stiffen relative to the stresses that drive the ice stream, and correspondingly whether $\tau \rightarrow \infty$.

\section{DISCUSSION AND CONCLUSIONS}

Comparison of the idealized features of the WIS stickslip behavior with the stick-slip motions exhibited by a simple, analogue model consisting of blocks and springs riding over a frictional substrate that follows a simple static and kinetic friction law shows that WIS can be considered to be a typical stick-slip system controlled by variations in the parameters describing its basal friction. Thus, the terminology originally chosen for the WIS motion by Bindschadler and others (2003b) is indeed appropriate. The strong influence of tides and the spring-to-neap cycle of their amplitude modulation (as solar and lunar components work against each other) is also featured in both the observed cycling of WIS and the simple model explored here. The role of a strong sticky spot (e.g. as identified on WIS by Wiens and others (2008) as Ice Rise A) is also mimicked by the simple model: the 'sticky block' in the model nucleates the stick-slip cycle, and the cycle's amplitude decays with distance from the sticky block. A specific feature that sets WIS apart from other ice streams that do not display stick-slip motion is the fact that WIS is slowing down (Bindschadler and Vornberger, 1998). This process is most likely associated with an increase in the basal friction of WIS. As our model results suggest, transitions from a steady slip to a stick-slip regime are possible when the ice stream encounters either an increase in basal friction or a decrease in overall flow speed. Thus, the stick-slip behavior of WIS may be a transitory feature associated with a slowing ice stream.

Observations of stick-slip events may provide important constraints on the basal conditions under an ice stream. Amplitude of displacements during stick events could be used to determine the bed yield stress (the static friction, $F_{\mathrm{S}}$, in our model). Analysis of GPS data from station B090 suggests that its yield stress is $\sim 0.5-1 \mathrm{kPa}$ (depending on the Young's modulus used in the model). Analysis of the slip displacements could provide estimates for basal shear during a slip event. To do that, PPP obtained from the GPS observations should be $\sim 30$ s or less.

\section{ACKNOWLEDGEMENTS}

We thank C. Schoof and two anonymous reviewers for catching errors in the original study and for constructive criticism and valuable suggestions for improving the manuscript. We also thank C. Hulbe for fruitful discussions. O.V.S. is supported by US National Science Foundation grant OPP-0632168.

\section{REFERENCES}

Afferrante, L. and M. Ciavarella. 2008. Thermo-elastic dynamic instability (TEDI) - a review of recent results. J. Eng. Math., 61 (24), 285-300.

Anandakrishnan, S., D.E. Voigt, R.B. Alley and M.A. King. 2003. Ice Stream D flow speed is strongly modulated by the tide beneath the Ross Ice Shelf. Geophys. Res. Lett., 30(7), 1361. (10.1029/2002GL016329.)

Bindschadler, R. and P. Vornberger. 1998. Changes in the West Antarctic ice sheet since 1963 from declassified satellite photography. Science, 279(5351), 689-692.

Bindschadler, R.A., M.A. King, R.B. Alley, S. Anandakrishnan and L. Padman. 2003a. Tidally controlled stick-slip discharge of a West Antarctic ice stream. Science, 301(5636), 1087-1089.

Bindschadler, R.A., P.L. Vornberger, M.A. King and L. Padman. 2003b. Tidally driven stick-slip motion in the mouth of Whillans Ice Stream, Antarctica. Ann. Glaciol., 36, 263-272.

Burridge, R. and L. Knopoff. 1967. Model and theoretical seismicity. Bull. Seismol. Soc. Am., 57(3), 341-371.

Carlson, J.M. and J.S. Langer. 1989. Mechanical model of an earthquake fault. Phys. Rev. A, 40(11), 6470-6484.

Chandler, D.M., R.I. Waller and W.G. Adam. 2005. Basal ice motion and deformation at the ice-sheet margin, West Greenland. Ann. Glaciol., 42, 67-70.

Danesi, S., S. Bannister and A. Morelli. 2007. Repeating earthquakes from rupture of an asperity under an Antarctic outlet glacier. Earth Planet. Sci. Lett., 253(1-2), 151-158.

De Baets, P., J. Degrieck, F. van de Velde and A.P. van Peteghem. 2000. Experimental verification of the mechanisms causing stick-slip motion originating from relative deceleration. Wear, 243(1-2), 48-59.

Gudmundsson, G.H. 2007. Tides and the flow of Rutford Ice Stream, West Antarctica. J. Geophys. Res., 112(F4), F04007. (10.1029/2006JF000731.)

Joughin, I. and 10 others. 2005. Continued deceleration of Whillans Ice Stream, West Antarctica. Geophys. Res. Lett., 32(22), L22501. (10.1029/2005GL024319.)

MacAyeal, D.R., M.A. King, J.N. Bassis and K.M. Brunt. 2007. All quiet on the seaward ice front? [Abstr. G33B-1231]. Eos, 88(54), Fall Meet. Suppl. 
Rice, J.R. and S.T. Tse. 1986. Dynamic motion of a single degree of freedom system following a rate and state dependent friction law. J. Geophys. Res., 91(B1), 521-530.

Rice, J.R., N. Lapusta and K. Ranjith. 2001. Rate and state dependent friction and the stability of sliding between elastically deformable solids. J. Mech. Phys. Solids, 49(9), 1865-1898.

Schoof, C. 2006a. A variational approach to ice stream flow. J. Fluid Mech., 556, 227-251.

Schoof, C. 2006b. Variational methods for glacier flow over plastic till. J. Fluid Mech., 555, 299-320.
Tulaczyk, S.M., B. Kamb and H.F. Engelhardt. 2000. Basal mechanics of Ice Stream B, West Antarctica. I. Till mechanics. J. Geophys. Res., 105(B1), 463-481.

Weertman, J. 2005. Slip event propagation direction in transition region of low surface slope. Ann. Glaciol., 40, 43-46.

Wiens, D.A., S. Anandakrishnan, J.P. Wineberry and M.A. King. 2008. Simultaneous teleseismic and geodetic observations of the stick-slip motion of an Antarctic ice stream. Nature, 453(7196), 770-774. 